\title{
Training changes processing of speech cues in older adults with hearing loss
}

\author{
Samira Anderson ${ }^{1,2+}$, Travis White-Schwoch ${ }^{1,2}$, Hee Jae Choi ${ }^{1,2}$ and Nina Kraus ${ }^{1,2,3,4,5 *}$ \\ ${ }^{1}$ Auditory Neuroscience Laboratory, Department of Communication Sciences and Disorders, Northwestern University, Evanston, IL, USA \\ ${ }^{2}$ Department of Communication Sciences, Northwestern University, Evanston, IL, USA \\ ${ }^{3}$ Institute for Neuroscience, Northwestern University, Evanston, IL, USA \\ ${ }^{4}$ Department of Neurobiology and Physiology, Northwestern University, Evanston, IL, USA \\ ${ }^{5}$ Department of Otolaryngology, Northwestern University, Evanston, IL, USA
}

\section{Edited by:}

Jonathan E. Peelle, Washington

University in St. Louis, USA

\section{Reviewed by:}

Preston E. Garraghty, Indiana

University, USA

Jonathan E. Peelle, Washington

University in St. Louis, USA

\section{*Correspondence:}

Nina Kraus, Auditory Neuroscience

Laboratory, Northwestern

University, 2240 Campus Drive,

Evanston, IL 60208, USA

e-mail:nkraus@northwestern.edu

www.brainvolts.northwestern.edu

${ }^{\dagger}$ Present address:

Samira Anderson, Department of Hearing and Speech Sciences,

University of Maryland, College

Park, USA
Aging results in a loss of sensory function, and the effects of hearing impairment can be especially devastating due to reduced communication ability. Older adults with hearing loss report that speech, especially in noisy backgrounds, is uncomfortably loud yet unclear. Hearing loss results in an unbalanced neural representation of speech: the slowly-varying envelope is enhanced, dominating representation in the auditory pathway and perceptual salience at the cost of the rapidly-varying fine structure. We hypothesized that older adults with hearing loss can be trained to compensate for these changes in central auditory processing through directed attention to behaviorally-relevant speech sounds. To that end, we evaluated the effects of auditory-cognitive training in older adults (ages 55-79) with normal hearing and hearing loss. After training, the auditory training group with hearing loss experienced a reduction in the neural representation of the speech envelope presented in noise, approaching levels observed in normal hearing older adults. No changes were noted in the control group. Importantly, changes in speech processing were accompanied by improvements in speech perception. Thus, central processing deficits associated with hearing loss may be partially remediated with training, resulting in real-life benefits for everyday communication.

Keywords: auditory plasticity, speech envelope, aging, hearing loss, speech perception, temporal fine structure, temporal coding

\section{INTRODUCTION}

Hearing loss is associated with reduced quality of life in older adults, affecting social and emotional well-being (Heine and Browning, 2002). The effects of hearing loss are especially noticeable in noisy backgrounds with multiple talkers (Dubno et al., 1984; Jin and Nelson, 2010). Many factors contribute to age-related deficits in speech-in-noise perception, including cochlear pathology (Dubno et al., 1984), impairments in central auditory processing (Phillips et al., 2000), and decreased cognitive resources (Pichora-Fuller, 2003; Wingfield et al., 2005; Peelle et al., 2010). Perceptual and neurophysiologic studies have demonstrated that central auditory processing is compromised by aging and hearing loss (Gordon-Salant et al., 2006; Clinard et al., 2010; Lister et al., 2011; Anderson et al., 2012). Hearing aid amplification improves audibility but cannot restore central auditory function (Tremblay et al., 2003). Training-driven neuroplasticity, which can partially reverse the effects of age-related deficits in temporal resolution in humans (Anderson et al., 2013c) and animals (de Villers-Sidani et al., 2010), may provide a means for treating abnormal central function processing associated with hearing loss.

One chief barrier to the wider use of amplification has been that hearing aids often make speech louder without improving clarity, especially in background noise (Johnson and Dillon, 2011). This phenomenon may arise from the effects of hearing loss on central processing of speech. Two acoustic aspects of speech, the slowly varying temporal envelope and the rapidly varying temporal fine structure (TFS), have been extensively studied (Ardoint et al., 2010; Hopkins and Moore, 2011). Although the envelope appears to be the dominant cue for understanding speech in quiet and in steady-state noise, both the envelope and TFS may play a role when listening to speech in fluctuating noise (Shannon et al., 1995; Moore, 2008). However, the role of TFS for understanding speech in fluctuating noise remains an area of debate (Oxenham and Simonson, 2009). In an animal model, sensorineural hearing loss is associated with enhanced envelope coding in the auditory nerve (Kale and Heinz, 2010) and midbrain (Anderson et al., 2013a). Perceptual studies also suggest exaggerated encoding of the envelope in humans with unilateral hearing loss (Moore et al., 1996) and potentially reduced ability to use TFS cues (Lorenzi et al., 2006). These effects might explain the hearing impaired listener's complaint that speech is loud yet unclear (Johnson and Dillon, 2011).

Because hearing aid amplification primarily addresses loss of audibility associated with cochlear pathology, there is a need to develop novel methods for counteracting the deleterious effects of hearing loss on central processing. Here, we assessed whether auditory-based cognitive training can be used to partially restore the imbalance of speech cue representation associated with exaggerated envelope encoding. Since both TFS (Sheft et al., 2008) and 
envelope (Swaminathan and Heinz, 2012) cues play an important role in consonant perception, we hypothesized that directed attention to the fast-changing consonant-vowel (CV) transition within an adaptive training paradigm results in reweighing of speech cue representation, such that the TFS becomes more salient. To test this hypothesis, we randomly assigned older adults with and without hearing loss to complete 8 weeks of computerized training. We evaluated subcortical representation of envelope and TFS cues before and after training in addition to speech perception in noise, auditory short-term memory, and auditory attention.

\section{MATERIALS AND METHODS PARTICIPANTS}

We recruited 58 (35 female) participants (ages 55-79) for a study examining training for speech-in-noise processing. Puretone audiometric thresholds were obtained bilaterally at octave intervals $0.125-8 \mathrm{kHz}$ and at 3 and $6 \mathrm{kHz}$. No left/right asymmetries or interaural click-evoked auditory brainstem response Wave $\mathrm{V}$ differences $(\geq 0.2 \mathrm{~ms}$ ) were noted. No participants had a history of neurologic conditions and all participants had normal IQs [ $\geq 85$ on the WASI (Zhu and Garcia, 1999)]. Participants provided informed consent for procedures that were approved by the Northwestern Institutional Review Board and were paid for their time.

Participants were divided into normal-hearing and hearingimpaired subgroups. The normal-hearing participants had hearing thresholds $\leq 25 \mathrm{~dB}$ HL through $6 \mathrm{kHz}$ and the participants with hearing loss had hearing thresholds $\leq 80 \mathrm{~dB}$ HL through $8 \mathrm{kHz}$. See Figure 1 for average thresholds in the normal-hearing and hearing-impaired groups. Participants from both hearing groups were randomly assigned to complete either auditorybased cognitive training or active control training (Smith et al., 2009). Both involved training on an in-home computer, $1 \mathrm{~h} /$ day, 5 days/week, for 8 weeks. The auditory training group completed Brain Fitness ${ }^{\mathrm{TM}}$ cognitive training (Posit Science Corporation, San Francisco, CA) consisting of six modules designed to increase the speed and accuracy of auditory processing: (1) time-order judgments of frequency-modulated sweeps, (2) discriminating between pairs of confusable syllables, (3) recognizing sequences of confusable syllables and words, (4) matching pairs of confusable syllables and words, (5) implementing sequences of commands, and (6) answering questions from stories (see Smith et al., 2009 for details). In the first module, an adaptively decreasing inter-stimulus interval challenges processing speed. In subsequent modules, focused attention is directed to adaptively expanding and contracting CV transitions. The overall goal of the training is to improve sensory function, based on the idea that an increase in the quality of neural information flowing through peripheral and central sensory systems may lead to better cognitive function (Schneider and Pichora-Fuller, 2000). Completion of training was verified through automated online logs. The active control group watched educational DVDs and completed multiple-choice questions about the content. See Figure 2 for a schematic of the experimental design. Training groups (and hearing subgroups) were matched for sex, age, hearing, click-evoked wave V brainstem latency, IQ, and test-retest intervals (Table 1).

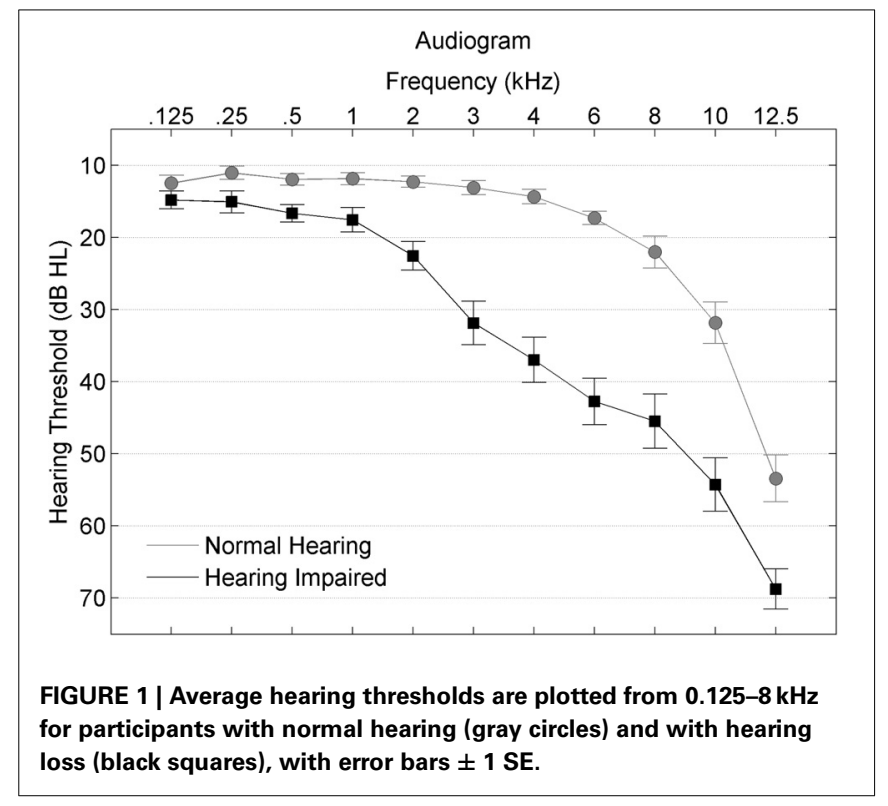

\section{BEHAVIORAL ASSESSMENTS}

\section{Perceptual-speech perception in noise}

The QuickSIN is a non-adaptive measure of speech perception in noise. Sentences are presented in a background of four-talker babble and the SNR decreases by $5 \mathrm{~dB}$ for each sentence. Participants receive a point for correctly repeating target words. The total number of points is subtracted from 25.5 to arrive at a final SNR loss [or the increase in SNR required to achieve 50\% correct performance compared to normal performance of $0 \mathrm{~dB}$ SNR; see Killion et al. (2004)].

\section{Cognitive}

Two subtests of the Woodcock-Johnson III Cognitive Test Battery (Woodcock et al., 2001) were used to obtain an age-normed cluster score for auditory short-term memory: Numbers Reversed and Memory for Words. The attention score was based on the sustained index of overall attention (visual and auditory) on the IVA+: Integrated Visual and Auditory Continuous Performance Test (BrainTrain, North Chesterfield, VA). Due to equipment malfunction, we are missing attention data from 3 participants in the auditory training group and 1 participant in the active control group.

\section{ELECTROPHYSIOLOGY Stimulus}

We duplicated the electrophysiologic methods previously used to document enhanced envelope coding in older adults with hearing loss (Anderson et al., 2013a), using the protocol that produced the greatest hearing loss effects. The stimulus was a 40-ms syllable [da] synthesized in a Klatt-based synthesizer (Klatt, 1980). It began with a 5-ms onset burst followed by a $\mathrm{CV}$ transition and was perceived as a full CV syllable although it lacked a steady-state vowel. After the initial onset burst, the fundamental frequency $\left(\mathrm{F}_{0}\right)$ of the stimulus rose linearly from 103 to $125 \mathrm{~Hz}$ while the formants shifted as follows: $\mathrm{F}_{1}: 220 \rightarrow$ $720 \mathrm{~Hz} ; \mathrm{F}_{2}: 1700 \rightarrow 1240 \mathrm{~Hz} ; \mathrm{F}_{3}: 2580 \rightarrow 2500 \mathrm{~Hz}$. The fourth 


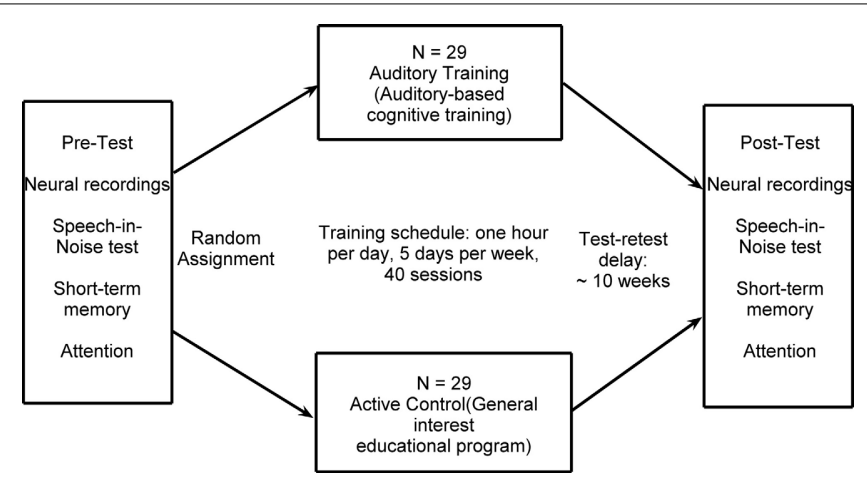

FIGURE 2 | A summary of the experimental design.

Table 1 | Means and SDs are provided for age, IQ, and hearing (PTA dB HL; average hearing threshold 0.5-4 kHz) for all participants in the Auditory Training and Active Control groups and the subgroups of normal hearing and hearing impaired.

\begin{tabular}{|c|c|c|c|c|c|c|}
\hline Mean $(S D)$ & \multicolumn{3}{|c|}{ Auditory training } & \multicolumn{3}{|c|}{ Active control } \\
\hline Age & $64.11(5.78)$ & $60.86(3.74)$ & $67.36(5.72)$ & $64.07(5.22)$ & $62.86(3.34)$ & $65.29(6.50)$ \\
\hline 10 & $118.43(12.15)$ & $117.00(12.30)$ & 119.36 (12.43) & $120.00(14.23)$ & 118.36 (14.28) & $122.56(14.61)$ \\
\hline Hearing (dB HL) & 20.64 (11.29) & $11.36(3.18)$ & $28.25(8.19)$ & $18.3(7.83)$ & $13.43(3.20)$ & $22.87(8.19)$ \\
\hline
\end{tabular}

The groups are matched on all listed variables (all p's $>0.10$ ).

$(3600 \mathrm{~Hz})$ and fifth $(4500 \mathrm{~Hz})$ formants remained constant for the duration of the stimulus. A spectrogram and Fourier transform of the stimulus waveform are presented in Figures 3A,B, respectively.

To partially equate for the effects of hearing loss, the [da] stimulus was individually amplified based on the National Acoustics Laboratory-Revised algorithm (NAL-R; Byrne and Dillon, 1986) using a custom program in MATLAB (The MathWorks, Inc., Natick, MA). The amplified presentation level did not exceed $90 \mathrm{~dB}$ SPL or the participants' loudness discomfort thresholds. We have previously found that the amplification procedure improves morphology without distorting spectral components of the response (Anderson et al., 2013b). In addition, although we found enhanced encoding in brainstem responses to both amplified and unamplified stimuli, the differences were clearest for the amplified stimuli (Anderson et al., 2013a); therefore, we expected to see the greatest treatment effects in response to the amplified stimuli. Repeated hearing tests verified that there were no changes in hearing after 8 weeks and therefore all participants received the same amplified stimulus before and after training.

\section{Recording}

The [da] was presented binaurally in pink noise at $+10 \mathrm{~dB}$ SNR. Stimuli were presented at $80 \mathrm{~dB}$ SPL for normal hearing listeners and amplified stimuli for participants with hearing loss were presented at an intensity of up to $90 \mathrm{~dB}$ SPL, with most stimuli in the range of $80-83 \mathrm{~dB}$ SPL. Stimuli were presented via the
Bio-Logic Navigator Pro System (Natus Medical, Inc., Mundelein, IL) at a rate of $10.9 \mathrm{~Hz}$ (inter-stimulus interval of $52 \mathrm{~ms}$ ) through electromagnetically shielded insert earphones (ER-3A, Etymotic Research, Elk Grove Village, IL). A vertical montage of four Ag$\mathrm{AgCl}$ electrodes ( $\mathrm{Cz}$ active, $\mathrm{Fpz}$ ground, earlobe references) was used with all contact impedances $<5 \mathrm{k} \Omega$. A criterion of $\pm 23 \mu \mathrm{V}$ was used for online artifact rejection. Two blocks of 3000 artifactfree sweeps were collected in each condition for each participant and averaged using an 85.3-ms window, including a 15.8-ms prestimulus period. Responses were sampled at $12 \mathrm{kHz}$ and were online bandpass filtered from 100 to $2000 \mathrm{~Hz}$ (Butterworth filter, $12 \mathrm{~dB}$ /octave, zero phase-shift) to minimize disruption by the low-frequency cortical response and to sample energy up to the phase-locking limits of the brainstem (Liu et al., 2006).

The [da] was presented in alternating polarities, allowing for the creation of responses comprised of both the sum and the difference of the two polarities (Campbell et al., 2012). When creating the summed frequency following response (FFR), the non-inverting envelope component of the response is enhanced while the inverting TFS component is minimized; conversely, for the subtracted responses the inverting TFS component is enhanced while the non-inverting envelope component is substantially reduced (Aiken and Picton, 2008). While this analysis is a somewhat different operationalization of envelope and TFS representation than is used in some other studies, the selection of measures of envelope and TFS representation is driven by the model organization and site of interest (Shamma and Lorenzi, 


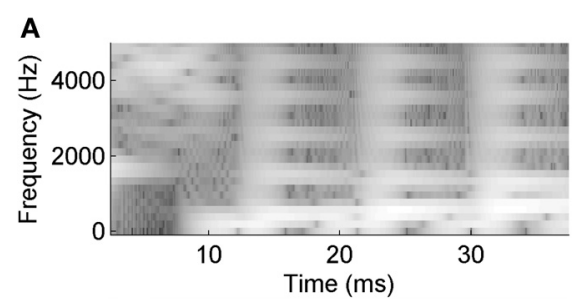

B

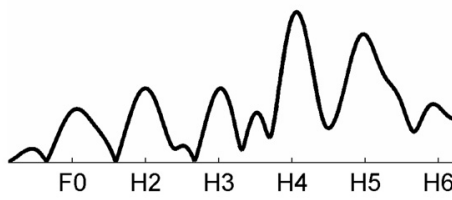

C

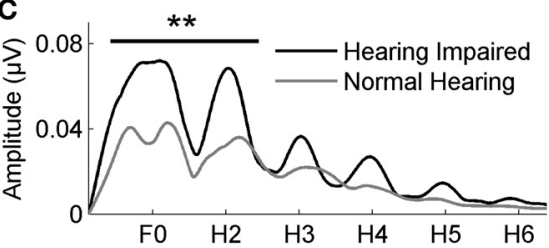

Envelope

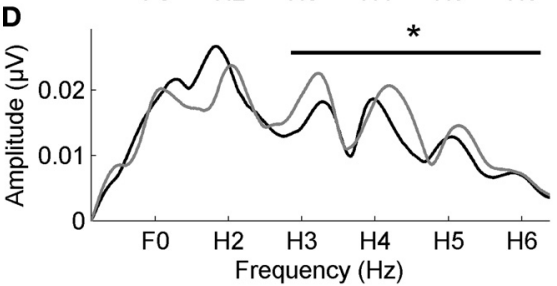

Fine Structure

FIGURE 3 | (A) The spectrogram of the 40-ms syllable [da]. Fast Fourier transforms were calculated from 20 to $42 \mathrm{~ms}$ for the stimulus (B) and in responses to the envelope (C) and the TFS (D) in noise. The average responses for the participants with normal hearing (gray) and with hearing loss (black) are displayed. The group with hearing loss has higher amplitudes in the envelope-dominated low frequencies $\left(\mathrm{F}_{0}-\mathrm{H}_{2}\right)$ in noise relative to the group with normal hearing (C); conversely, the group with normal hearing has higher amplitudes in the fine structure dominated higher frequencies $\left(\mathrm{H}_{3}-\mathrm{H}_{6}\right)$ than the group with hearing loss (D). ${ }^{*} p<0.05$, ${ }^{* *} p<0.01$

2013), and adding/subtracted brainstem responses to alternating polarities is a typical method used in analyzing human FFRs (Aiken and Picton, 2008; Gockel et al., 2011; Anderson et al., 2013a). Nevertheless, we note that the relationship between envelope/TFS representation reflected in the FFR, perceptual measures, and auditory nerve coding remains an avenue for future research. Spectral amplitudes were calculated using fast Fourier transforms (FFTs) over $60 \mathrm{~Hz}$ bins around the frequencies of interest, which included the fundamental frequency $\left(\mathrm{F}_{0}\right)$ and its integer harmonics. The time region chosen for this calculation was $20-42 \mathrm{~ms}$, corresponding to the most periodic time region of the FFR.

\section{STATISTICAL ANALYSIS}

A Multivariate Analysis of Variance (MANOVA) was used to assess hearing group differences in the envelope and TFS. The $\mathrm{F}_{0}$ and $\mathrm{H}_{2}$ amplitudes from the added polarities (henceforth $\mathrm{F}_{0 \mathrm{ADD}}-$ $\mathrm{H}_{2 \mathrm{ADD}}$ ) were entered as dependent variables in the MANOVA to represent the envelope, as these lower frequency spectral peaks dominate the envelope-following FFR. The $\mathrm{H}_{3}-\mathrm{H}_{6}$ amplitudes from the subtracted polarities $\left(\mathrm{H}_{3 S U B}-\mathrm{H}_{6 \mathrm{SUB}}\right)$ were entered as dependent variables to represent the TFS, which is more prominent in relatively higher frequencies in the FFR. A repeated measures ANOVA was used to compare envelope-dominated $\left(\mathrm{F}_{0 A D D}-\mathrm{H}_{2 \mathrm{ADD}}\right)$ and TFS-dominated $\left(\mathrm{H}_{3 \mathrm{SUB}}-\mathrm{H}_{6 \mathrm{SUB}}\right)$ frequency encoding, QuickSIN SNR scores, and memory and attention measures before and after training.

\section{RESULTS}

\section{EFFECTS OF HEARING LOSS ON ENCODING SPEECH ENVELOPE/TFS}

The combined participants with hearing loss from both groups had greater representation of the envelope $\left(\mathrm{F}_{0 \mathrm{ADD}}\right.$ and $\left.\mathrm{H}_{2 \mathrm{ADD}}\right)$ than participants with normal hearing $\left[F_{(1,57)}=7.218, p=\right.$ 0.002] (Figure 3C), replicating a previous study demonstrating greater subcortical representation of the envelope in response to speech in noise in older adults with hearing loss (Anderson et al., 2013a). In contrast to the Anderson et al. study (2013a), however, we also found reduced representation of the TFS $\left(\mathrm{H}_{3 S U B}-\mathrm{H}_{6 \mathrm{SUB}}\right)$ in the older adults with hearing loss $\left[F_{(1,57)}=3.066, p=0.024\right]$ (Figure 3D). The current study comprised 58 participants vs. the 30 participants in the previous study; therefore, the new finding of significant differences in TFS representation in the current study may be attributed to increased power and is in fact consistent with Henry and Heinz (2012), who found a reduction in TFS coding in noise in auditory nerve fibers of chinchillas with noise-induced hearing loss.

\section{TRAINING: NEUROPHYSIOLOGICAL CHANGES}

In the group with hearing loss, we found a training group $\times$ test session interaction $\left[F_{(1,30)}=4.351, p=0.023\right]$, with a significant reduction in envelope encoding $\left(\mathrm{F}_{0 \mathrm{ADD}}\right.$ to $\left.\mathrm{H}_{2 \mathrm{ADD}}\right)$ occurring within the auditory training group $\left[F_{(1,14)}=3.843, p=0.049\right]$ but not the active control group $\left[F_{(1,14)}=0.381, p=0.691\right]$ (see Figures 4A,B). We also analyzed the pre and post data of the groups with normal hearing and found no training group $\times$ test session interaction $\left[F_{(1,27)}=0.803, p=0.459\right]$ and no changes in either group (all $p$ 's $>0.1$ ) (see Figures 4C,D). Although there was no training group $\times$ hearing group $\times$ test session interaction $\left[F_{(1,57)}=2.239, p=0.117\right]$, there was a hearing group $\times$ test session interaction in the auditory training group $\left[F_{(1,29)}=\right.$ 3.573, $p=0.043]$ that was not present in the active control group $\left[F_{(1,29)}=0.136, p=0.874\right]$ (see Figures 4E,F), suggesting that the auditory training effect was specific to the participants with hearing loss. Representation of the TFS $\left(\mathrm{H}_{3 \mathrm{SUB}}-\mathrm{H}_{6 \mathrm{SUB}}\right)$ did not change for either hearing impaired or normal hearing participants of either training group (all $p$ 's $>0.1$ ) (Figure 5). Figures 6, 7 display mean $\mathrm{F}_{0 \mathrm{ADD}}$ and $\mathrm{H}_{2 \mathrm{ADD}}$ and mean $\mathrm{H}_{3 \mathrm{SUB}}-$ $\mathrm{H}_{6 \mathrm{SUB}}$ amplitudes, respectively, for individual participants. It is evident from these figures that individuals with hearing loss have greater variability than individuals with normal hearing.

\section{TRAINING: BEHAVIORAL CHANGES}

Summary: There were significant training-induced changes in speech-in-noise perception, memory, and attention across hearing groups in the auditory training group. The training effects 

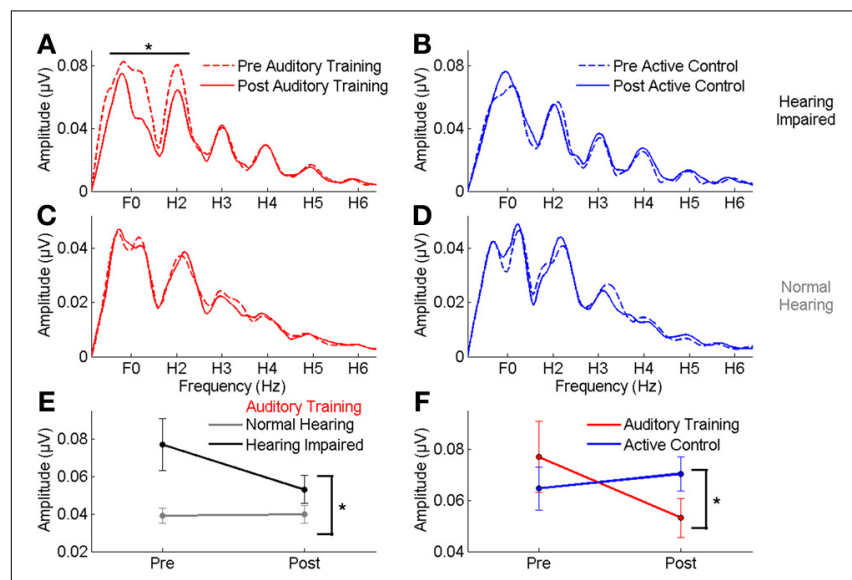

FIGURE 4 | (A,B) A comparison of pre- (dotted lines) and post-training responses (solid lines) to speech in noise to the envelope $\left(\mathrm{F}_{0}-\mathrm{H}_{2}\right)$ in the auditory training (red) and active control (blue) groups with hearing loss, demonstrating a significant reduction in response to the envelope in the auditory training group. (C,D) No reduction was seen in response to the envelope in either group with normal hearing. (E) A significant hearing $x$ session interaction was noted in the auditory training group, demonstrating that the change was specific to the participants with hearing loss. (F) A significant group $\times$ session interaction in the groups with hearing loss indicating a reduction in the representation of the $\mathrm{F}_{0}$ in the auditory training group only. ${ }^{*} p<0.05$. Error bars: $\pm 1 \mathrm{SE}$.
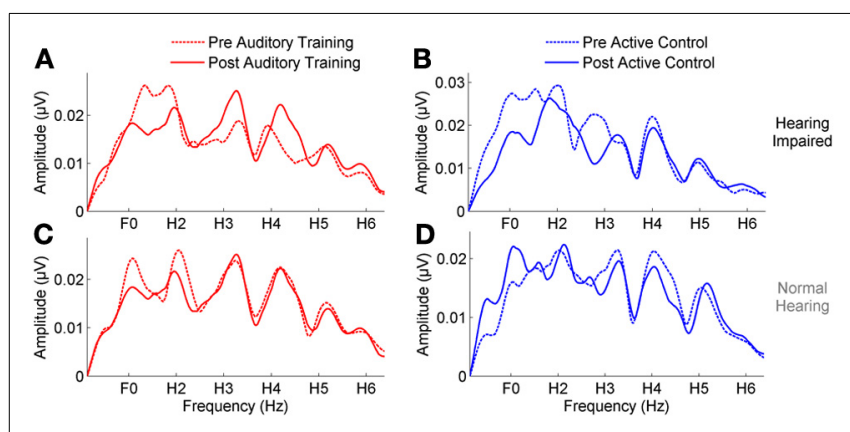

FIGURE 5 | (A,B) A comparison of pre- (dotted lines) and post-training responses (solid lines) to speech in noise to the fine structure $\left(\mathrm{H}_{3}-\mathrm{H}_{6}\right)$ in the auditory training (red) and active control (blue) groups with hearing loss, demonstrating no change in either group. (C,D) No change was seen in response to the fine structure in either group with normal hearing.

specific to hearing status varied depending on the task. The improvement in speech-in-noise performance was specific to the hearing impaired group, the memory change was found only in the normal hearing group, and attention improved in both groups. There were no corresponding changes in the active control group.

For speech-in-noise performance (QuickSIN), there was a significant training group $\times$ test session interaction $\left[F_{(1,57)}=5.191, p=0.027\right]$, with improvement noted in the auditory training group $\left[F_{(1,28)}=13.394, p=0.001\right]$ but not in the active control group $\left[F_{(1,28)}=1.678, p=0.206\right]$. This change was largely driven by the improvement in performance in the auditory training group with hearing loss $\left[F_{(1,14)}=12.220, p=\right.$

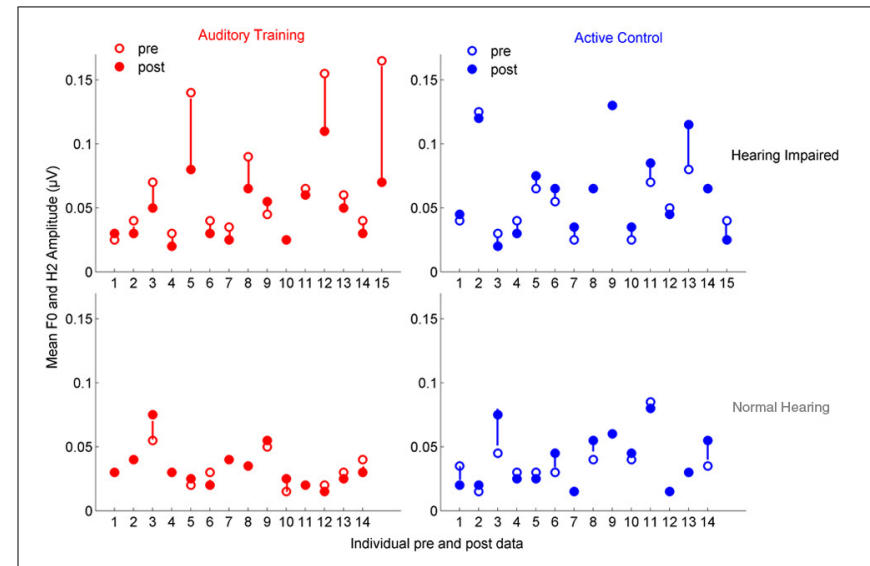

FIGURE 6 | Mean $\mathrm{F}_{0 A D D}$ and $\mathrm{H}_{2 A D D}$ amplitudes are displayed for individual pre- (open circles) and post-data (closed circles) for the auditory training (red) and active control (blue) groups. Visual observation of the data reveals that there is greater pre-training variability in both groups with hearing loss and in the degree of change in the auditory training group with hearing loss.

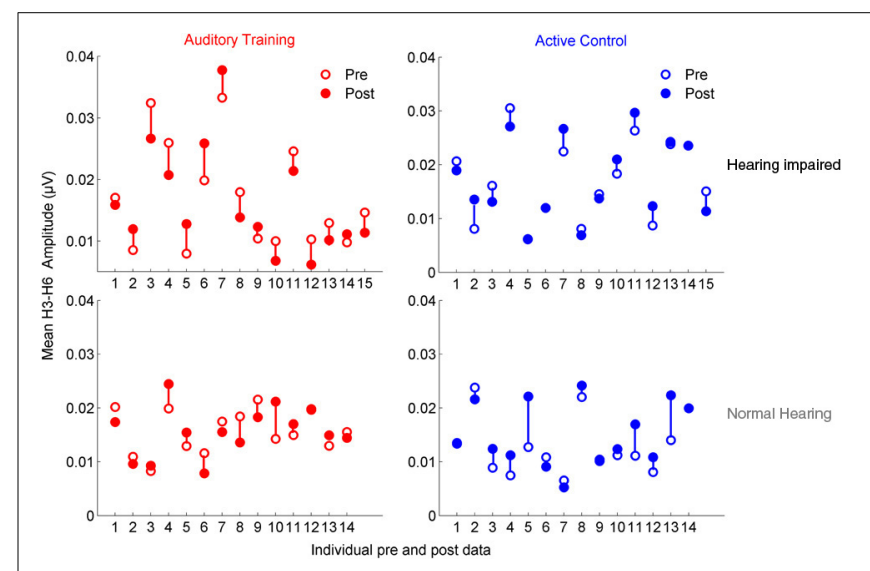

FIGURE 7 | Mean $\mathrm{H}_{3}$ SUB and $\mathrm{H}_{6 \text { SUB }}$ amplitudes are displayed for individual pre- (open circles) and post-data (closed circles) for the auditory training (red) and active control (blue) groups. Similar to the data for the envelope, the data demonstrates greater variability in both groups with hearing loss for pre-test data, but there is no systematic change with training as was found for the envelope.

$0.004]$; the improvement in the normal-hearing group was not significant $\left[F_{(1,14)}=3.041, p=0.105\right]$. Neither hearing group in the active control group changed with training (all $p$ 's $>0.1$ ). There was a decrease in the QuickSIN of $1.22 \mathrm{~dB}$ in the auditory training group with hearing loss-given four lists, this number is below the $1.9 \mathrm{~dB}$ necessary for a critical difference between conditions with an 95\% confidence interval (Killion et al., 2004). However, a $1 \mathrm{~dB}$ decrease in SNR corresponds to approximately a $10 \%$ increase in word recognition-a difference that is likely to be noticeable to the listener (Middelweerd et al., 1990). It should also be noted that the participants with hearing loss did not have more than a mild SNR loss-less than $7 \mathrm{~dB}$ - so greater gains might be expected from individuals with greater deficits. 
Similarly, for short-term memory there was a significant training group $\times$ test session interaction $\left[F_{(1,57)}=6.042, p=0.017\right]$, with improvements in the auditory training group $\left[F_{(1,28)}=\right.$ 9.800, $p=0.004]$ but not the active control group $\left[F_{(1,28)}=\right.$ $0.158, p=0.694]$. For memory, however, the changes were only significant in the group with normal hearing $\left[F_{(1,28)}=7.648\right.$, $p=0.016]$ but not in the group with hearing loss $\left[F_{(1,28)}=\right.$ $2.630, p=0.127]$ and neither hearing group in the active control group changed (all $p$ 's $>0.1$ ).

Finally, for attention, there was a significant training group $\times$ test session interaction $\left[F_{(1,50)}=3.765, p=0.043\right]$, with improvements in the auditory training group $\left[F_{(1,25)}=\right.$ 17.941, $p<0.001]$ but not the active control group $\left[F_{(1,24)}=\right.$ $0.623, p=0.438]$. In this case, there were significant improvements for both the subgroups with normal hearing $\left[F_{(1,11)}=\right.$ 8.182, $p=0.016]$ and with hearing loss of the auditory training group $\left[F_{(1,12)}=9.339, p=0.009\right]$. Again, there were no changes for members of either hearing subgroup of the active control group (all $p$ 's $>0.1$ ). See Figure 8 for interaction plots of behavioral changes. Please refer to Table 2 for means and standard deviations of pre- and post-training changes in behavioral measures for the normal hearing and hearing impaired participants of the auditory training and active control groups.

\section{DISCUSSION}

Here we show that the imbalance in neurophysiological processing of speech cues associated with hearing loss is malleable and reversible with training in older adults. The results are summarized as follows: first, compared to normal hearing individuals, older adults with hearing loss have excessively large envelope encoding of speech in noise. Second, training that targets the $\mathrm{CV}$ transition reduces envelope representation in individuals with hearing loss to levels in line with those of normal-hearing individuals. Finally, the training-induced changes in neurophysiology are accompanied by gains in speech-in-noise perception, attention, and short-term memory, although it should be noted that the change in speech-in-noise perception was modest, and would not be considered clinically significant (Killion et al., 2004). Nevertheless, we note that an improvement of $1 \mathrm{~dB}$ SNR corresponds to approximately $10 \%$ word intelligibility in noise (Middelweerd et al., 1990), so the changes may be perceived as beneficial to the listener.

Our results confirm previous findings of exaggerated representation of envelope cues in animal and human models of sensorineural hearing loss (Kale and Heinz, 2010; Henry and Heinz, 2012; Anderson et al., 2013a), providing a possible explanation for the observation that the hearing impaired listener perceives speech as loud but unclear (Jin and Nelson, 2010). At present, the

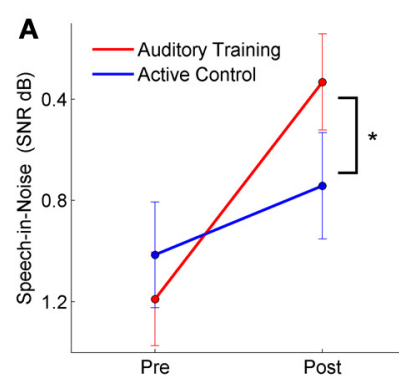

FIGURE 8 | Pre and post-training perceptual and cognitive scores for participants with both normal hearing and hearing loss (group $x$ session interactions). The auditory training group improved in
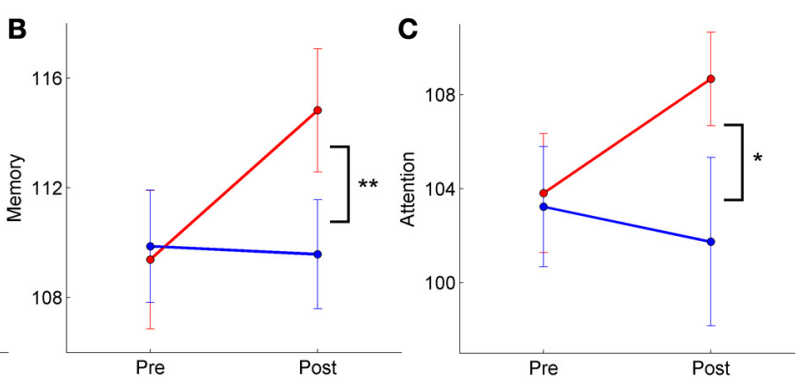

speech-in-noise perception (A), memory (B), and attention (C), whereas there were no changes in the active control group. ${ }^{*} p<0.05,{ }^{* *} p<0.01$. Error bars: $\pm 1 \mathrm{SE}$.

Table 2 | Means and SDs are provided for pre- and post-test scores for speech-in-noise perception, auditory short-term memory and attention for the Auditory Training and Active Control groups, including the subgroups of participants with normal hearing and with hearing loss.

\begin{tabular}{|c|c|c|c|c|c|c|c|}
\hline \multirow[t]{2}{*}{ Mean (SD) } & \multirow[t]{2}{*}{ Session } & \multicolumn{3}{|c|}{ Auditory training } & \multicolumn{3}{|c|}{ Active control } \\
\hline & & $\begin{array}{l}\text { Total } \\
(N=29)\end{array}$ & $\begin{array}{l}\text { Normal hearing } \\
(N=14)\end{array}$ & $\begin{array}{l}\text { Hearing impaired } \\
(N=15)\end{array}$ & $\begin{array}{l}\text { Total } \\
(N=29)\end{array}$ & $\begin{array}{l}\text { Normal hearing } \\
(N=14)\end{array}$ & $\begin{array}{l}\text { Hearing impaired } \\
(N=15)\end{array}$ \\
\hline \multirow{2}{*}{$\begin{array}{l}\text { Speech-in-noise } \\
\text { perception }\end{array}$} & Pre & $1.51(1.44)$ & $0.72(0.82)$ & $2.04(1.38)$ & $1.13(1.16)$ & $0.93(1.26)$ & $1.31(1.07)$ \\
\hline & Post & $0.65(1.27)$ & $0.25(1.08)$ & $0.84(1.32)$ & $0.91(1.22)$ & $0.63(1.14)$ & $1.18(1.27)$ \\
\hline \multirow[t]{2}{*}{ Memory } & Pre & $09.28(14.24)$ & $104.71(12.77)$ & 113.53 (14.62) & 110.38 (11.65) & 113.53 (14.62) & $109.80(7.72)$ \\
\hline & Post & 114.24 (13.08) & 111.43 (14.32) & 116.57 (11.67) & $109.72(11.34)$ & 116.57 (11.67) & 108.07 (10.87) \\
\hline \multirow[t]{2}{*}{ Attention } & Pre & $103.48(14.47)$ & $99.08(17.44)$ & 107.00 (10.96) & 103.62 (15.48) & $100.00(16.51)$ & $107.11(12.27)$ \\
\hline & Post & $108.25(11.81)$ & 104.50 (12.38) & $112.00(10.30)$ & 99.35 (23.61) & $100.46(24.90)$ & $105.37(24.24)$ \\
\hline
\end{tabular}


mechanisms underlying this exaggerated representation in auditory brainstem are unknown; however, evidence from auditory nerve suggests a peripheral etiology arising from reduced outer hair cell compression in cases of mild to moderate hearing loss and from inner hair cell damage (steeper input-output functions) in cases of moderate to severe hearing loss (Kale and Heinz, 2010). The fact that envelope coding changed with training suggests that there may also be a top-down central gain effect resulting from auditory deprivation (Munro and Blount, 2009). However, there was no change in TFS coding in the training group. The effects of hearing loss on envelope and TFS representation are a function of complex interactions among cochlear function, stimulus presentation level, and SNR (Henry and Heinz, 2012). One possibility is that a change in TFS coding was too subtle to be observed at the single SNR we used. Another important consideration is the nature of the training: all of the training stimuli were presented in quiet, not in noise. It is possible that this presentation technique favored envelope coding, which is maladaptively enhanced even in quiet in listeners with cochlear hearing loss (Anderson et al., 2013a). Further investigation to establish the effects of hearing loss at different presentation levels and SNRs is warranted, as a better understanding of the mechanisms underlying abnormal stimulus encoding will help guide future treatment efforts.

The results of our study contrast with those of two previous studies that found training-induced increases in envelope coding in normal-hearing young adults using different types of training: pitch discrimination training (Carcagno and Plack, 2011) and recognition of speech presented in babble and other challenging conditions (Song et al., 2012). The key difference between these studies and ours is that our study population included individuals with hearing impairment. Given the differences between normal-hearing and hearing-impaired listeners in performance on perceptual tasks, neurophysiological encoding of sound, and reliance on cognitive mechanisms for speech intelligibility (Lorenzi et al., 2006; Anderson et al., 2013a; Humes et al., 2013), it is not necessarily surprising that there are different effects of auditory training. Another important difference is the training itself. The training in our study directed attention to fast-changing sounds in high memory load situations and occurred in quiet. It may be that training on recognition of speech in noise, such as in the Song et al. study, produces more robust gains in speech-in-noise perception and different outcomes when comparing pre and post envelope and TFS coding. As another example, FFR neural representation of the $\mathrm{F}_{0}$ is correlated with behavioral pitch discrimination, (Krishnan et al., 2010; Marmel et al., 2013); therefore, enhanced neural representation of cues contributing to the perception of pitch may underlie the traininginduced gains in pitch discrimination found by Carcagno and Plack (2011). Thus, outcomes may vary depending on the type of training and the targeted population.

We propose, therefore, that the training effects in this study were influenced by neural mechanisms specific to older adults with hearing loss. Both aging (Turner et al., 2005; Schatteman et al., 2008) and hearing loss (Vale and Sanes, 2002; Dong et al., 2009) appear to cause an imbalance in excitatory and inhibitory function, likely affecting stimulus encoding. However, this imbalance is at least partially reversed in animal models of auditory training (de Villers-Sidani et al., 2010) and acoustic experience (Turner et al., 2013). Although we are unable to verify these effects in humans, we speculate that the training-induced changes were facilitated by alterations in the balance of neurotransmitter levels to allow for precise encoding of subtle CV differences. That said, just as it is difficult to disambiguate between peripheral (i.e., outer hair cell loss and peripheral neuropathy) vs. central mechanisms of hearing loss, it is difficult to say with certainty which mechanisms were targeted by training.

Our training was designed to strengthen sensory function through attention to meaningful sound; specifically, focusing attention on CV transitions in speech may drive top-down modulation, which occurred in five of the six training modules. Animal models have demonstrated that directed attention to behaviorally-relevant stimuli is necessary for neural and behavioral plasticity (Fritz et al., 2005, 2007). In our study, we found that neural response changes were accompanied by improvements in attention. A functional connection between prefrontal and auditory cortices provides a basis for efferent activation during difficult tasks that require focused attention (Raizada and Poldrack, 2007). A tentative connection has also been suggested between prefrontal cortex and auditory brainstem based on fMRI studies (Roelfsema et al., 2010). Although our methodology does not allow us to draw similar conclusions, we propose that rapid TFS cues may become more salient when sensory and cognitive demands drive a prefrontal-brainstem connection to adjust subcortical encoding of these cues.

A number of questions remain unanswered. We did not include anyone in our study who wore hearing aids, because hearing aids themselves may induce plasticity in auditory processing (Munro et al., 2007; Munro and Merrett, 2013). Therefore, the use of amplification itself can be considered a form of training. Hornickel et al. (2012) demonstrated that assistive listening devices engender improved trial-to-trial consistency in brainstem firing, presumably by directing attention to a more robust and noise-free representation of the stimulus. Future work should compare the neural effects of amplification alone vs. amplification plus training. In addition, more work should be done to determine the persistence of training effects and the time course of learning. A number of approaches were employed in our training protocol, involving strictly bottom-up discrimination training and combined memory and perceptual training exercises. It would be important to identify the aspects of training that were primarily responsible for engendering plasticity. We note that there is a great deal of individual variability in the participants with hearing loss, both in terms of pre-training spectral amplitudes and in the degree of change. In future work, it will be important to identify sources of variability, and the factors that contribute to success in individuals.

Finally, although neither envelope nor TFS representation changed in the normal hearing group, this group did experience changes in attention and short-term memory. Therefore, we assume the normal-hearing participants experienced traininginduced biological changes that were not observed using the current envelope/TFS technique. The lack of observed improvement in memory in the group with hearing loss may be due to the auditory nature of our assessment measure. Although the 
presentation level was adjusted to a comfortable volume for each participant, it may be that peripheral hearing loss limited performance, even in the post-training session, given the known effects of hearing loss on verbal short-term memory (McCoy et al., 2005; Verhaegen et al., 2013). The speech-in-noise perception scores (QuickSIN) of normal hearing groups would be considered clinically normal-less than $3 \mathrm{~dB}$ SNR loss (Killion et al., 2004), and so the lack of improvement in this group probably stems from a ceiling effect. Further investigation is needed to determine the mechanisms of training-induced improvements in individuals with normal hearing.

These results have implications for management of hearing loss problems in older adults. Although provision of audibility is a necessary foundation for the treatment of hearing difficulties, we assert that auditory training is just as important. The combination of amplification and auditory training may lead to improved speech clarity in noise without distortion from overamplification.

\section{ACKNOWLEDGMENTS}

We thank Trent Nicol, Karen Chan, and Alexandra Parbery-Clark for their critical reviews of the manuscript. Supported by the NIH (R01 DC010016) and the Knowles Hearing Center.

\section{REFERENCES}

Aiken, S. J., and Picton, T. W. (2008). Envelope and spectral frequency-following responses to vowel sounds. Hear. Res. 245, 35-47. doi: 10.1016/j.heares.2008. 08.004

Anderson, S., Parbery-Clark, A., White-Schwoch, T., Drehobl, S., and Kraus, N. (2013a). Effects of hearing loss on the subcortical representation of speech cues. J. Acoust. Soc. Am. 133, 3030-3038. doi: 10.1121/1.4799804

Anderson, S., Parbery-Clark, A., White-Schwoch, T., and Kraus, N. (2013b). Auditory brainstem response to complex sounds predicts self-reported speechin-noise performance. J. Speech Lang. Hear. Res. 56, 31-43. doi: 10.1044/10924388(2012/12-0043)

Anderson, S., White-Schwoch, T., Parbery-Clark, A., and Kraus, N. (2013c). Reversal of age-related neural timing delays with training. Proc. Natl. Acad. Sci. U.S.A. 110, 4357-4362. doi: 10.1073/pnas.1213555110

Anderson, S., Parbery-Clark, A., White-Schwoch, T., and Kraus, N. (2012). Aging affects neural precision of speech encoding. J. Neurosci. 32, 14156-14164. doi: 10.1523/JNEUROSCI.2176-12.2012

Ardoint, M., Sheft, S., Fleuriot, P., Garnier, S., and Lorenzi, C. (2010). Perception of temporal fine-structure cues in speech with minimal envelope cues for listeners with mild-to-moderate hearing loss. Int. J. Audiol. 49, 823-831. doi: 10.3109/14992027.2010.492402

Byrne, D., and Dillon, H. (1986). The National Acoustic Laboratories' (NAL) new procedure for selecting the gain and frequency response of a hearing aid. Ear Hear. 7, 257-265. doi: 10.1097/00003446-198608000-00007

Campbell, T., Kerlin, J., Bishop, C., and Miller, L. (2012). Methods to eliminate stimulus transduction artifact from insert earphones during electroencephalography. Ear Hear. 33, 144-150. doi: 10.1097/AUD.0b013e3182280353

Carcagno, S., and Plack, C. (2011). Subcortical plasticity following perceptual learning in a pitch discrimination task. J. Assoc. Res. Otolaryngol. 12, 89-100. doi: 10.1007/s10162-010-0236-1

Clinard, C. G., Tremblay, K. L., and Krishnan, A. R. (2010). Aging alters the perception and physiological representation of frequency: evidence from human frequency-following response recordings. Hear. Res. 264, 48-55. doi: 10.1016/j.heares.2009.11.010

de Villers-Sidani, E., Alzghoul, L., Zhou, X., Simpson, K. L., Lin, R. C. S., and Merzenich, M. M. (2010). Recovery of functional and structural age-related changes in the rat primary auditory cortex with operant training. Proc. Natl. Acad. Sci. U.S.A. 107, 13900-13905. doi: 10.1073/pnas.1007885107

Dong, S., Mulders, W., Rodger, J., and Robertson, D. (2009). Changes in neuronal activity and gene expression in guinea-pig auditory brainstem after unilateral partial hearing loss. Neuroscience 159, 1164-1174. doi: 10.1016/j.neuroscience.2009.01.043

Dubno, J. R., Dirks, D. D., and Morgan, D. E. (1984). Effects of age and mild hearing loss on speech recognition in noise. J. Acoust. Soc. Am. 76, 87-96. doi: $10.1121 / 1.391011$

Fritz, J., Elhilali, M., and Shamma, S. (2005). Active listening: task-dependent plasticity of spectrotemporal receptive fields in primary auditory cortex. Hear. Res. 206, 159-176. doi: 10.1016/j.heares.2005.01.015

Fritz, J. B., Elhilali, M., David, S. V., and Shamma, S. A. (2007). Does attention play a role in dynamic receptive field adaptation to changing acoustic salience in A1? Hear. Res. 229, 186-203. doi: 10.1016/j.heares.2007.01.009

Gockel, H., Carlyon, R., Mehta, A., and Plack, C. (2011). The frequency following response (FFR) may reflect pitch-bearing information but is not a direct representation of pitch. J. Assoc. Res. Otolaryngol. 12, 767-782. doi: 10.1007/s10162-011-0284-1

Gordon-Salant, S., Yeni-Komshian, G. H., Fitzgibbons, P. J., and Barrett, J. (2006). Age-related differences in identification and discrimination of temporal cues in speech segments. J. Acoust. Soc. Am. 119, 2455-2466. doi: 10.1121/ 1.2171527

Heine, C., and Browning, C. J. (2002). Communication and psychosocial consequences of sensory loss in older adults: overview and rehabilitation directions. Disabil. Rehabil. 24, 763-773. doi: 10.1080/09638280210129162

Henry, K. S., and Heinz, M. G. (2012). Diminished temporal coding with sensorineural hearing loss emerges in background noise. Nat. Neurosci. 15, 1362-1364. doi: 10.1038/nn.3216

Hopkins, K., and Moore, B. C. J. (2011). The effects of age and cochlear hearing loss on temporal fine structure sensitivity, frequency selectivity, and speech reception in noise. J. Acoust. Soc. Am. 130, 334-349. doi: 10.1121/ 1.3585848

Hornickel, J., Zecker, S. G., Bradlow, A. R., and Kraus, N. (2012). Assistive listening devices drive neuroplasticity in children with dyslexia. Proc. Natl. Acad. Sci. U.S.A. 109, 16731-16736. doi: 10.1073/pnas.1206628109

Humes, L. E., Kidd, G. R., and Lentz, J. J. (2013). Auditory and cognitive factors underlying individual differences in aided speech-understanding among older adults. Front. Syst. Neurosci. 7:55. doi: 10.3389/fnsys.2013.00055

Jin, S. H., and Nelson, P. B. (2010). Interrupted speech perception: the effects of hearing sensitivity and frequency resolution. J. Acoust. Soc. Am. 128, 881. doi: $10.1121 / 1.3458851$

Johnson, E. E., and Dillon, H. (2011). A comparison of gain for adults from generic hearing aid prescriptive methods: impacts on predicted loudness, frequency bandwidth, and speech intelligibility. J. Am. Acad. Audiol. 22, 441-459. doi: 10.3766/jaaa.22.7.5

Kale, S., and Heinz, M. (2010). Envelope coding in auditory nerve fibers following noise-induced hearing loss. J. Assoc. Res. Otolaryngol. 11, 657-673. doi: 10.1007/s10162-010-0223-6

Killion, M., Niquette, P., Gudmundsen, G., Revit, L., and Banerjee, S. (2004). Development of a quick speech-in-noise test for measuring signal-to-noise ratio loss in normal-hearing and hearing-impaired listeners. J. Acoust. Soc. Am. 116, 2395-2405. doi: 10.1121/1.1784440

Klatt, D. (1980). Software for a cascade/parallel formant synthesizer. J. Acoust. Soc. Am. 67, 971-995. doi: 10.1121/1.383940

Krishnan, A., Bidelman, G., and Gandour, J. T. (2010). Neural representation of pitch salience in the human brainstem revealed by psychophysical and electrophysiological indices. Hear. Res. 268, 60-66. doi: 10.1016/j.heares. 2010.04.016

Lister, J. J., Roberts, R. A., and Lister, F. L. (2011). An adaptive clinical test of temporal resolution: age effects. Int. J. Audiol. 50, 367-374. doi: $10.3109 / 14992027.2010 .551218$

Liu, L.-F., Palmer, A. R., and Wallace, M. N. (2006). Phase-locked responses to pure tones in the inferior colliculus. J. Neurophysiol. 95, 1926-1935. doi: 10.1152/jn.00497.2005

Lorenzi, C., Gilbert, G., Carn, H., Garnier, S., and Moore, B. C. J. (2006). Speech perception problems of the hearing impaired reflect inability to use temporal fine structure. Proc. Natl. Acad. Sci. U.S.A. 103, 18866-18869. doi: 10.1073/pnas.0607364103

Marmel, F., Linley, D., Carlyon, R. P., Gockel, H. E., Hopkins, K., and Plack, C. J. (2013). Subcortical neural synchrony and absolute thresholds predict frequency discrimination independently. J. Assoc. Res. Otolaryngol. 14, 757-766. doi: $10.1007 / \mathrm{s} 10162-013-0402-3$ 
McCoy, S. L., Tun, P. A., Cox, L. C., Colangelo, M., Stewart, R. A., and Wingfield, A. (2005). Hearing loss and perceptual effort: downstream effects on older adults' memory for speech. Q. J. Exp. Psychol. A 58, 22-33. doi: 10.1080/02724980443000151

Middelweerd, M., Festen, J., and Plomp, R. (1990). Difficulties with speech intelligibility in noise in spite of a normal pure-tone audiogram. Int. J. Audiol. 29, 1-7. doi: 10.3109/00206099009081640

Moore, B. C. J. (2008). The role of temporal fine structure processing in pitch perception, masking, and speech perception for normal-hearing and hearing-impaired people. J. Assoc. Res. Otolaryngol. 9, 399-406. doi: 10.1007/s10162-008-0143-x

Moore, B. C. J., Wojtczak, M., and Vickers, D. A. (1996). Effect of loudness recruitment on the perception of amplitude modulation. J. Acoust. Soc. Am. 100, 481-489. doi: 10.1121/1.415861

Munro, K. J., and Blount, J. (2009). Adaptive plasticity in brainstem of adult listeners following earplug-induced deprivation. J. Acoust. Soc. Am. 126, 568-571. doi: $10.1121 / 1.3161829$

Munro, K. J., and Merrett, J. F. (2013). Brainstem plasticity and modified loudness following short-term use of hearing aids. J. Acoust. Soc. Am. 133, 343-349. doi: $10.1121 / 1.4770234$

Munro, K. J., Pisareva, N. Y., Parker, D. J., and Purdy, S. C. (2007). Asymmetry in the auditory brainstem response following experience of monaural amplification. Neuroreport 18, 1871-1874. doi: 10.1097/WNR.0b013e3282f1b003

Oxenham, A. J., and Simonson, A. M. (2009). Masking release for low- and highpass-filtered speech in the presence of noise and single-talker interference. J. Acoust. Soc. Am. 125, 457-468. doi: 10.1121/1.3021299

Peelle, J. E., Troiani, V., Wingfield, A., and Grossman, M. (2010). Neural processing during older adults' comprehension of spoken sentences: age differences in resource allocation and connectivity. Cereb. Cortex 20, 773-782. doi: 10.1093/cercor/bhp142

Phillips, S. L., Gordon-Salant, S., Fitzgibbons, P. J., and Yeni-Komshian, G. (2000) Frequency and temporal resolution in elderly listeners with good and poor word recognition. J. Speech Lang. Hear. Res. 43, 217-228.

Pichora-Fuller, M. K. (2003). Cognitive aging and auditory information processing. Int. J. Audiol. 42, 26-32. doi: 10.3109/14992020309074641

Raizada, R. D. S., and Poldrack, R. A. (2007). Challenge-driven attention: interacting frontal and brainstem systems. Front. Hum. Neurosci. 1:3. doi: 10.3389/neuro.09.003.2007

Roelfsema, P. R., van Ooyen, A., and Watanabe, T. (2010). Perceptual learning rules based on reinforcers and attention. Trends Cogn. Sci. 14, 64. doi: 10.1016/j.tics.2009.11.005

Schatteman, T. A., Hughes, L. F., and Caspary, D. M. (2008). Aged-related loss of temporal processing: altered responses to amplitude modulated tones in rat dorsal cochlear nucleus. Neuroscience 154, 329-337. doi: 10.1016/j.neuroscience.2008.02.025

Schneider, B., and Pichora-Fuller, K. (2000). "Implications of perceptual deterioration for cognitive aging research," in The Handbook of Aging and Cognition, eds F. M. I. Craik and T. A. Salthouse (Mahwah, NJ: Lawrence Erlbaum Associates), 155-219.

Shamma, S., and Lorenzi, C. (2013). On the balance of envelope and temporal fine structure in the encoding of speech in the early auditory system. J. Acoust. Soc. Am. 133, 2818. doi: 10.1121/1.4795783

Shannon, R. V., Zeng, F.-G., Kamath, V., Wygonski, J., and Ekelid, M. (1995). Speech recognition with primarily temporal clues. Science 270, 303. doi: $10.1126 /$ science.270.5234.303
Sheft, S., Ardoint, M., and Lorenzi, C. (2008). Speech identification based on temporal fine structure cues. J. Acoust. Soc. Am. 124, 562-575. doi: $10.1121 / 1.2918540$

Smith, G. E., Housen, P., Yaffe, K., Ruff, R., Kennison, R. F., Mahncke, H. W., et al. (2009). A cognitive training program based on principles of brain plasticity: results from the improvement in memory with plasticity-based adaptive cognitive training (IMPACT) study. J. Am. Geriatr. Soc. 57, 594-603. doi: 10.1111/j.1532-5415.2008.02167.x

Song, J. H., Skoe, E., Banai, K., and Kraus, N. (2012). Training to improve hearing speech in noise: biological mechanisms. Cereb. Cortex 22, 1180-1190. doi: $10.1093 /$ cercor/bhr196

Swaminathan, J., and Heinz, M. G. (2012). Psychophysiological analyses demonstrate the importance of neural envelope coding for speech perception in noise. J. Neurosci. 32, 1747-1756. doi: 10.1523/JNEUROSCI.449311.2012

Tremblay, K., Piskosz, M., and Souza., P. (2003). Effects of age and age-related hearing loss on the neural representation of speech cues. Clin. Neurophysiol. 114, 1332-1343. doi: 10.1016/S1388-2457(03)00114-7

Turner, J. G., Hughes, L. F., and Caspary, D. M. (2005). Affects of aging on receptive fields in rat primary auditory cortex layer V neurons. J. Neurophysiol. 94, 2738-2747. doi: 10.1152/jn.00362.2005

Turner, J. G., Parrish, J. L., Zuiderveld, L., Darr, S., Hughes, L. F., Caspary, D. M., et al. (2013). Acoustic experience alters the aged auditory system. Ear Hear. 34, 151-159. doi: 10.1097/AUD.0b013e318269ca5b

Vale, C., and Sanes, D. H. (2002). The effect of bilateral deafness on excitatory and inhibitory synaptic strength in the inferior colliculus. Eur. J. Neurosci. 16, 2394-2404. doi: 10.1046/j.1460-9568.2002.02302.x

Verhaegen, C., Collette, F., and Majerus, S. (2013). The impact of aging and hearing status on verbal short-term memory. Neuropsychol. Dev. Cogn. B Aging Neuropsychol. Cogn. doi: 10.1080/13825585.2013.832725. [Epub ahead of print].

Wingfield, A., Tun, P. A., and McCoy, S. L. (2005). Hearing loss in older adulthood. Curr. Dir. Psychol. Sci. 14, 144-148. doi: 10.1111/j.0963-7214.2005. 00356.x

Woodcock, R. W., McGrew, K. S., and Mather, N. (2001). Woodcock-Johnson III Tests of Cognitive Abilities Itasca, IL: Riverside Publishing.

Zhu, J., and Garcia, E. (1999). The Wechsler Abbreviated Scale of Intelligence (WASI). New York, NY: Psychological Corporation.

Conflict of Interest Statement: The authors declare that the research was conducted in the absence of any commercial or financial relationships that could be construed as a potential conflict of interest.

Received: 02 August 2013; accepted: 11 November 2013; published online: 28 November 2013.

Citation: Anderson S, White-Schwoch T, Choi HJ and Kraus $N$ (2013) Training changes processing of speech cues in older adults with hearing loss. Front. Syst. Neurosci. 7:97. doi: 10.3389/fnsys.2013.00097

This article was submitted to the journal Frontiers in Systems Neuroscience.

Copyright (c) 2013 Anderson, White-Schwoch, Choi and Kraus. This is an openaccess article distributed under the terms of the Creative Commons Attribution License (CC BY). The use, distribution or reproduction in other forums is permitted, provided the original author(s) or licensor are credited and that the original publication in this journal is cited, in accordance with accepted academic practice. No use, distribution or reproduction is permitted which does not comply with these terms. 